\title{
The evolution of mass loaded supernova remnants
}

\section{Temperature dependent mass injection rates}

\author{
J. M. Pittard ${ }^{1}$, S. J. Arthur ${ }^{2}$, J. E. Dyson ${ }^{1}$, S. A. E. G. Falle ${ }^{3}$, T. W. Hartquist ${ }^{1}$, M. I. Knight ${ }^{1}$, and M. Pexton ${ }^{3}$ \\ 1 Department of Physics \& Astronomy, The University of Leeds, Woodhouse Lane, Leeds LS2 9JT, UK \\ 2 Instituto de Astronomia, UNAM, Campus Morelia, Apartado Postal 3-72, 58090 Morelia, Michoacan, Mexico \\ 3 Department of Applied Mathematics, The University of Leeds, Woodhouse Lane, Leeds LS2 9JT, UK
}

Received 21 August 2002 / Accepted 4 February 2003

\begin{abstract}
We investigate the evolution of spherically symmetric supernova remnants in which mass loading takes place due to conductively driven evaporation of embedded clouds. Numerical simulations reveal significant differences between the evolution of conductively mass loaded and the ablatively mass loaded remnants studied in Paper I. A main difference is the way in which conductive mass loading is extinguished at fairly early times, once the interior temperature of the remnant falls below $\sim 10^{7} \mathrm{~K}$. Thus, at late times remnants that ablatively mass load are dominated by loaded mass and thermal energy, while those that conductively mass load are dominated by swept-up mass and kinetic energy. Simple approximations to the remnant evolution, complementary to those in Paper I, are given.
\end{abstract}

Key words. ISM: kinematics and dynamics - ISM: supernova remnants - galaxies: ISM

\section{Introduction}

The evolution and properties of supernova remnants are fundamental to many areas of astrophysical research. Self-similar solutions for various stages of the evolution have been obtained for various assumptions, as have unified solutions which link these together (see Cioffi et al. 1988; Truelove \& McKee 1999). The self-similar and unified solutions are complemented by a wide range of numerical investigations. Amongst the problems examined numerically are explosions in plane-stratified media (e.g. Falle \& Garlick 1982; Arthur \& Falle 1991, 1993), the evolution of SNRs inside pre-existing wind-driven cavities (Franco et al. 1991), the influence of hydrodynamic instabilities (e.g. Chevalier \& Blondin 1995), and applications to the broad emission line regions in active galactic nuclei (e.g. Terlevich et al. 1992; Pittard et al. 2001).

In most investigations of supernova remnant evolution, the surrounding medium has been assumed to have a smooth density distribution. However, the interstellar medium is known to be multi-phase (e.g. McKee \& Ostriker 1977), and there is clear observational evidence of engulfed clouds within the supernova remnant N63A (Chu et al. 1999; Warren et al. 2002). As it is widely known, the injection of mass from such cool clouds into the interior of a supernova remnant will affect its behaviour and structure.

If the clouds are assumed to be continuously distributed, similarity solutions describing the evolution of adiabatic mass

Send offprint requests to: J. M. Pittard,

e-mail: jmp@ast.leeds.ac.uk loaded supernova remnants can be derived for a specific functional dependence of the mass injection. Previous work has focussed on cases in which one mass injection process is dominant over others. McKee \& Ostriker (1977), Chièze \& Lazareff (1981), and White \& Long (1991) each investigated the evolution under the assumption that cloud destruction is due to conductive evaporation. Alternatively, Dyson \& Hartquist (1987) treated hydrodynamic ablation as the dominant process.

A small number of papers based on numerical simulations of mass loaded supernova remnants also exist in the literature. Cowie et al. (1981) included the dynamics of the clouds and found that warm clouds are swept towards the shock front and are rapidly destroyed, while cold clouds are more evenly distributed and have longer lifetimes. When energy losses become important, this behaviour leads to the highest densities in the remnant occuring over the outer half radius. In contrast, when there is no mass loading, a thin dense shell forms at the forward shock. Arthur \& Henney (1996) studied the effects of mass loading by hydrodynamic ablation on supernova remnants evolving inside cavities evacuated by the stellar winds of the progenitor stars. They showed that the extra mass injected by embedded clumps was capable of producing the excess soft $\mathrm{X}$-ray emission seen in some bubbles in the Large Magellanic Cloud.

The range of a supernova remnant is relevant to a number of important areas of astronomy. It can affect the efficiency of sequential star formation and the global dynamics and structures of starburst superwinds where many remnants overlap. The superwind in the starburst galaxy M 82 must be mass loaded to 
account for the observed X-ray emission (Suchkov et al. 1996). Since the range and radiative energy losses of a remnant are affected by mass loading, it is therefore desirable to have approximations which describe remnant evolution and range in clumpy media.

Analogous approximations already exist for smooth media (cf. Truelove \& McKee 1999, and references therein). In a recent paper (Dyson et al. 2002, hereafter Paper I), the first steps towards this goal were taken with the derivation of range approximations for cases in which mass injection occurs at a constant rate, $q$, or at a rate which depends on the flow Mach number relative to the clump (Hartquist et al. 1986). A mass loading rate of $q M^{4 / 3}$ was used for subsonic flow, where $M$ is the Mach number of the flow. A constant mass loading rate $q$ was used where the flow is supersonic. This prescription simulates mass stripping from the clump by hydrodynamic ablation.

In this companion paper we consider mass injection from clouds into the hot remnant interior by conductive evaporation (cf. Cowie \& McKee 1977; McKee \& Cowie 1977). This process is potentially important in any astrophysical system where a hot tenuous phase coexists with a colder denser phase (see McKee \& Cowie 1977 for some examples), though it can be suppressed by magnetic fields. In many systems (including supernova remnants) the hot phase flows past the clouds, and they will be subject also to ablation. Unfortunately, we currently lack even basic models of this combined interaction, so it is difficult to know whether one process dominates the other, or if one process limits the other. For example, one could imagine a scenario whereby ablation drives the initial mass loss, increasing the effective surface area of the cloud (e.g. by forming a tail), at which point conduction takes over and leads to efficient mixing. In evolving objects such as supernova remnants, one process may dominate at early evolutionary stages, while the other dominates at later stages. Given these potential complications and our lack of knowledge about them, as a first approach we treat each process individually.

The outline of our paper is as follows. In Sect. 2 we outline our assumptions and our numerical code; in Sect. 3 we present some of our results; in Sect. 4 we present simple approximations to these results; and in Sect. 5 we summarize and conclude.

\section{The calculations}

As initial conditions, we take freely-expanding cold ejecta at constant density (typically $10^{4}$ times the ambient density, $n_{0}$ ), and with a linear velocity profile. The maximum ejecta speed is $4000 \mathrm{~km} \mathrm{~s}^{-1}$, which together with the radius of the ejecta defines the initial time. We performed extensive tests with different density ratios to confirm that our chosen ratio is large enough not to influence the resulting evolution. The calculations were performed using an adaptive grid hydrodynamic code (see e.g. Falle \& Komissarov 1996, 1998), and we have further verified that the resolution employed is high enough to give robust results for the global quantities considered in this paper.

All calculations were performed in spherical symmetry, with an ejecta mass of $10 M_{\odot}$ and a kinetic energy of $10^{51} \mathrm{ergs}$ (the effect of different progenitor masses and explosion energies is commented on in Sect. 5). The assumption that the energy is entirely kinetic for the initial conditions is somewhat different to that in Paper I, but it does not affect the resulting evolution (see also Gull 1973 and Cowie et al. 1981). We modelled the intercloud ambient medium as a warm plasma of temperature $10^{4} \mathrm{~K}$. Cooling appropriate for an optically thin plasma of solar composition and in collisional ionization equilibrium was included. We assumed that cooling below $10^{4} \mathrm{~K}$ is balanced by photoionization heating from the diffuse field and for that reason impose a temperature floor of $10^{4} \mathrm{~K}$. For simplicity we did not include magnetic fields or shock precursors, and also did not consider conduction between the hot remnant interior and cold neighbouring gas.

The rate of mass evaporation from embedded clouds is dependent on many factors (cf. Cowie \& McKee 1977), including the temperature of the hot phase, the cloud radius, and the presence of magnetic fields. However, motions of the clouds are generally not large enough to change the evaporation rates, and Cowie \& Songalia (1977) noted that nonspherical clouds may be treated in an approximate way by adopting half the largest dimension as the radius of the cloud. The evaporative mass-loss rate from a single cloud (Cowie \& McKee 1977) is

$\dot{m}=4 \pi a^{2} n T^{1 / 2}\left(k \mu m_{\mathrm{H}}\right)^{1 / 2} \phi F\left(\sigma_{0}\right)$,

where $a$ is the cloud radius, $n$ and $T$ are the density and temperature of the surrounding hot phase (i.e. the interior of the remnant), $\mu m_{\mathrm{H}}$ is the mean mass per particle, and $\phi$ is an efficiency factor which encapsulates the magnetic field strength, its configuration, the cloud geometry, etc. $(\phi=0$ corresponds to no evaporation, $\phi \approx 1$ to evaporation in the absence of magnetic fields - e.g. $\phi=1.1$ for a plasma with equal electron and ion temperatures and cosmic abundances). $F\left(\sigma_{0}\right)$ is a function defined in Eqs. (61) and (62) of Cowie \& McKee (1977). In the classical limit, $F\left(\sigma_{0}\right)=2 \sigma_{0}$, where the saturation parameter, $\sigma_{0}$, is defined as

$\sigma_{0}=\left(\frac{T}{1.54 \times 10^{7}}\right)^{2} \frac{1}{n a_{\mathrm{pc}} \phi}$.

Here $a_{\mathrm{pc}}=a /(1 \mathrm{pc})$ is the radius of the cloud in parsecs. The onset of saturation occurs when $\sigma_{0}$ is of order unity, varying between $1.95(\phi \ll 1)$ and $1.08(\phi=1)$ for the examples given in Cowie \& McKee (1977). In the classical limit, $\dot{m} \propto T^{5 / 2}$ since $F\left(\sigma_{0}\right) \propto \sigma_{0}$ (cf. Fig. 4 in Cowie \& McKee 1977). If saturated, $F\left(\sigma_{0}\right) \propto \sigma_{0}^{\beta}$, where $\beta=\left(1+M_{\mathrm{s}}^{2}\right) /\left(6+M_{\mathrm{s}}^{2}\right)$, and $M_{\mathrm{s}}$ (the Mach number of the saturated zone) is related to $\phi$ by

$M_{\mathrm{s}}\left(1+\frac{1}{5} M_{\mathrm{s}}^{2}\right)=2 \phi$.

Since $\phi$ is unlikely to exceed unity by a substantial amount, and has a lower limit of zero, the likely range of $\beta$ is $1 / 6 \leq \beta \leq 3 / 8$. Hence in the saturated limit $\dot{m} \propto T^{\alpha}$ where $5 / 6 \leq \alpha \leq 5 / 4$.

Since the onset of saturation is dependent on the radius of the cloud for a specified hot phase, larger clouds will tend to evaporate in the classical limit, while the evaporation of mass from smaller clouds will tend towards saturation. As the temperature and density of the remnant interior evolves, the radius of clouds which are just at the onset of saturation will 
also change. Moreover, we expect the distribution of clouds to evolve with time, as the timescale for the smaller clouds to completely evaporate is shorter than the equivalent timescale for larger clouds and in many cases the evolutionary timescale of the remnant. Further, we may expect some differences in the physical make-up of clouds of differing radii (e.g. the distribution of cloud mass in the cold core and its warmer skin - cf. McKee \& Ostriker 1977). Finally, a full treatment also requires specifying the number of clouds per unit volume, which is likely to be dependent on the local environment (e.g. the cloud spectrum inside a starburst superwind is likely to be somewhat different to that in our local ISM). McKee \& Ostriker (1977) argued that mass loading in the Galactic ISM would be dominated by the smaller clouds.

Addressing all of these issues is beyond the scope of the present paper, so we choose instead the following simplification. We assume that we can specify a temperature for the onset of saturation, $T_{\mathrm{sat}}$, which is an average over both the cloud distribution and time. We adopt $T_{\text {sat }}=10^{7} \mathrm{~K}$ for all of our calculations. Given the uncertainties introduced by this assumption (and those in the original work of Cowie \& McKee 1977) it seems unrealistic to specify a complicated dependence for the mass evaporation rate per unit volume above $T=T_{\text {sat }}$, and we therefore assume that it is independent of $T$. Tests with other values of $T_{\text {sat }}$ have shown that our results only become sensitive to the value of $T_{\text {sat }}$ when $T_{\text {sat }}<10^{7} \sqrt{n_{0}} \mathrm{~K}\left(n_{0}\left(\mathrm{~cm}^{-3}\right)\right.$ is the intercloud ambient number density). Hence our results with $n_{0} \leq 1 \mathrm{~cm}^{-3}$ are robust (since a lower value of $T_{\text {sat }}$ implies that most of the mass loading is from a population of tiny clouds $\left(a_{\mathrm{pc}}<0.42 / \phi\right)$ whereas the smallest cloud radii in models of the ISM by McKee \& Ostriker, 1977, are $\sim 0.38 \mathrm{pc}$ ). On the other hand, for $n_{0}>1 \mathrm{~cm}^{-3}$, our results are dependent on our chosen value of $T_{\text {sat }}$. However, as $T_{\text {sat }}$ will depend on details such as clump lifetimes and number distributions, which are problem specific parameters, we simply note that our results begin to suffer from loss of generality in this regime.

Our final assumptions are: i) that the intercloud spacing is small compared to the scale over which the properties of the remnant varies (so that a continuous mass source term can be used), ii) that injection takes place at zero velocity relative to the global flow, and iii) that the injected gas has zero internal energy. The full set of gas dynamic equations is then

$\frac{\partial \rho}{\partial t}+\frac{1}{r^{2}} \frac{\partial}{\partial r} r^{2} \rho u=q$

$\frac{\partial \rho u}{\partial t}+\frac{1}{r^{2}} \frac{\partial}{\partial r} r^{2}\left(p+\rho u^{2}\right)=\frac{2 p}{r}$,

$\frac{\partial e}{\partial t}+\frac{1}{r^{2}} \frac{\partial}{\partial r}\left[r^{2} u(e+p)\right]=-L$,

where the symbols $\rho, u$, and $p$ have their usual meanings, $e=p /(\gamma-1)+0.5 \rho u^{2}$ is the total energy, and $L$ is the radiative cooling term. $q$ is the mass loading rate per unit volume and time.

As in Paper I, we define a fiducial mass loading rate

$q_{0}=\frac{6 \rho_{0}}{5 t_{\mathrm{sf}}}$ which gives the mass flux through the blast wave divided by the remnant volume at the onset of thin shell formation for Sedov expansion. Here, $\rho_{0}$ is the intercloud ambient density and $t_{\mathrm{sf}}$ is the timescale for thin shell formation for a remnant propagating into a uniform ambient medium given by Franco et al. (1994),

$t_{\mathrm{sf}}=2.87 \times 10^{4} E_{51}^{3 / 14} n_{0}^{-4 / 7} \mathrm{yrs}$,

where $10^{51} E_{51}$ is the explosion energy. In these units, $q_{0}=$ $1.32 \times 10^{-36} E_{51}^{-3 / 14} n_{0}^{11 / 7}$ (with mean mass per particle, $\mu=0.6$ ).

Following the above discussion, we define the mass loading rate due to conduction as

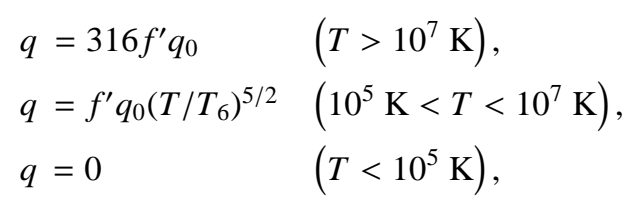

where $T_{6}=10^{6} \mathrm{~K}$ (e.g. Cowie et al. 1981). Thin shell formation occurs when the temperature immediately behind the leading shock is about $10^{6} \mathrm{~K}$. At low temperatures, mass loading is so weak that the precise temperature cutoff is unimportant. Defining $f^{\prime}$ as we have in Eq. (9) ensures that mass loading starts to influence the dynamics and evolution of the remnant if $f^{\prime} \sim 1$. To be more specific, low values of $f^{\prime}$ mean that mass loading will not dominate in the remnant before the time of onset of thin shell formation, while $f^{\prime}>1$ indicates that the evaporated mass becomes important in the remnant before $t_{\mathrm{sf}}$ is reached. Readers who refer to Paper I will note that in that paper we defined a parameter $f$ which is somewhat analogous to $f^{\prime}$. In an ablatively mass loaded remnant the ablated mass is comparable to the swept-up mass at time $t_{\mathrm{sf}}$ if $f \sim 1$. However, under the assumptions we have made, in very young remnants in which the temperature is everywhere above $10^{7} \mathrm{~K}$, $f \sim 316 f^{\prime}$ for the mass loading rate in an ablatively loaded remnant to be comparable to that in a conductively loaded remnant for which $E_{0}$ and $n_{0}$ are the same.

$f^{\prime}$ is physically related to the number density of clouds and the rate at which mass is evaporated from these. However, from Eq. (13) of Cowie et al. (1981), and since $q_{0} \propto E_{51}^{-3 / 14} n_{0}^{11 / 7}$, we find that

$f^{\prime} \propto \phi a_{\mathrm{pc}} N_{\mathrm{c}} E_{51}^{3 / 14} n_{0}^{-11 / 7}$,

where $N_{\mathrm{c}}$ is the number density of clouds. Thus we might expect real remnants to be better described by small $f^{\prime}$ when they evolve in environments with high ambient densities, and larger $f^{\prime}$ when they evolve in environments with low ambient densities. We note that if the distribution of clouds evolve with time, $f^{\prime}$ itself may evolve with time, though such an effect is not investigated in the models presented in this paper.

We note here, that differences exist between this work and Paper I. In the calculations presented here, mass loading is permitted throughout the remnant, including the ejecta material, whereas in Paper I it is switched off in the ejecta. This causes differences when most of the mass loading occurs before the remnant has swept up much intercloud mass (i.e. when $f^{\prime}$ and $n_{0}$ are large). A further difference is the assumed temperature of the ambient gas. In Paper I, a temperature of $100 \mathrm{~K}$ 
was used, whereas here we use $10^{4} \mathrm{~K}$. This will have negligible consequences until near the merger of the remnant with the surrounding gas. We note that in the specific case of superwind generation, merger may take place with gas at even higher temperatures.

We have computed models with $f^{\prime}=0,0.316,3.16,31.6$, 316 , and ambient densities $n_{0}=0.01,0.1,1,10,100 \mathrm{~cm}^{-3}$. Although there is an indication that high values of $f^{\prime}$ will be favoured when remnants evolve in surroundings with low ambient densities, $f^{\prime}$ also scales with the number density of clouds, and thus could be completely independent of the density of the intercloud medium. Hence we explored $\left(f^{\prime}, n_{0}\right)$ parameter space.

The remnants are evolved until the blast front degenerates into an acoustic wave. We adopted the same definitions for the ends of the free expansion and Quasi-Sedov-Taylor phases as given in Paper I. These are respectively when the internal energy reaches $60 \%$ of the initial energy (FE) and when $50 \%$ of the initial energy has been radiated (QST). For $f^{\prime} \lesssim 3.16$, the mass loading dominates in the remnant at times before the onset of thin shell formation, $t_{\mathrm{sf}}$, though is always decreasing in importance at this point (i.e. the ratio of loaded mass to sweptup mass is falling). For $f^{\prime}>3.16$ the mass loading also remains dominant at the time of the end of the QST stage, $t_{\mathrm{QST}}$. Finally, as in Paper I, we note the caveat that we are considering a purely general case and that, in practice, $f^{\prime}$ is determined by the actual physical situation being studied. We therefore ignore details such as clump lifetimes and spatial distributions, which only introduce additional, problem specific, parameters.

\section{Results}

We note that all of the results presented in this paper and Paper I are for a particular set of explosion parameters, namely the canonical values $E=10^{51} \mathrm{erg}$ and $M=10 M_{\odot}$. Where results for hydrodynamic ablation or constant mass loading are presented these have been calculated with the same code as used in the conductive mass loading cases but for the mass loading prescription given in Paper I.

\subsection{Remnant expansion speeds and ranges}

In Fig. 1 we show the remnant expansion speed and radius as functions of time for calculations with zero mass loading $\left(f^{\prime}=\right.$ 0 ), substantial mass loading $\left(f^{\prime}=3.16\right)$, and high mass loading $\left(f^{\prime}=31.6\right)$, and for ambient densities of $n_{0}=0.01 \mathrm{~cm}^{-3}$ and $n_{0}=100 \mathrm{~cm}^{-3}$. Mass loading causes a reduction in expansion velocity and range at intermediate ages, but there is convergence in speeds and ranges at later times, regardless of the value of $f^{\prime}$ or the ambient density. This is in contrast to remnants which mass load through ablation (Paper I) where the reductions in expansion speed and remnant range persist right through to the point of merger with the ambient medium.

This dynamical behaviour can be understood as follows. At early times, the highest temperatures are in the shocked ISM material so mass loading is highest there. By conservation of momentum, the addition of mass to this gas reduces its velocity and also has the effect of increasing the pressure (by conservation of energy). This causes the shocked material to brake harder and leads to an overall reduction in the expansion velocity of the remnant. Once the ejecta have completely thermalized, the highest temperatures are in the shocked ejecta material and most of the mass is added here, increasing the density and pressure in this gas. At this stage the rate of decrease of the expansion velocity is less in the conductively mass loaded remnants than in the zero mass loading case because of the higher pressure in the remnant interior. As the blast wave decelerates, the postshock temperatures and hence the conductive mass loading rates decrease, so during this stage the swept-up mass becomes more important. Eventually, the swept-up mass dominates the dynamics in the postshock region and the remnant evolution tends towards the zero mass loading case.

\subsection{Mass loading history}

Figure 2 shows the ratio of injected to swept-up mass as a function of time for various values of $f^{\prime}$ and ambient densities $n_{0}$. The top panels show the evolution when mass is injected through hydrodynamic ablation, while the bottom panels show the results obtained when mass injection occurs by conductive evaporation. It is immediately obvious from this figure that these mass loading prescriptions yield vastly different behaviour.

When mass loading occurs through ablation, the injected mass becomes increasingly dominant over the swept up mass as time progresses. The limit of the ablative mass loading is a constant mass loading rate $q$ (corresponding to supersonic flow throughout the remnant), so that the injected mass increases as $\dot{M}_{\text {load }} \approx 4 \pi R(t)^{3} q / 3$, whereas the rate of increase of the sweptup mass is $\dot{M}_{\text {swept }}=4 \pi R(t)^{2} \rho_{0} \mathrm{~d} R(t) / \mathrm{d} t$, so that

$\frac{\dot{M}_{\text {load }}}{\dot{M}_{\text {swept }}} \propto \frac{R(t) q}{\dot{R}(t) \rho_{0}} \propto f^{\prime} t / t_{\mathrm{sf}}$,

where we have used Eq. (7) and approximated the remnant expansion speed as $\dot{R}(t) \propto R(t) / t$. Hence, as the ratio of the rates increases approximately linearly with time, we expect that the ratio of the injected to swept-up mass will also increase approximately linearly with time. This is indeed the behaviour that we see in Figs. 2a, b. Furthermore, for a given $f^{\prime}$, we expect that the ratio of the loaded mass $\left(M_{\text {load }}\right)$ to the swept-up mass $\left(M_{\text {swept }}\right)$ as a function of time (in units of $\left.t_{\text {sf }}\right)$ will be independent of $n_{0}$, as is indeed seen (Fig. 2b). With reference to Eq. (10), however, "mathematical" independence of $n_{0}$ (at constant $f^{\prime}$ ) does not imply "physical" independence (i.e. keeping constant the other physically meaningful parameters, like $\left.\phi, a_{\mathrm{pc}}, N_{\mathrm{c}}, E_{51}\right)$.

When mass loading occurs via conductively-driven evaporation, on the other hand, the behaviour of $M_{\text {load }} / M_{\text {swept }}$ as a function of $t$ is quite different (cf. Figs. 2a, c). Although the injected mass exceeds the swept-up mass at intermediate times, as the remnant ages the rate of mass loading becomes negligible, and the swept-up mass gradually dominates. In Figs. 2c, $\mathrm{d}$, the swept-up mass is, in all cases, greater than the injected mass at the point when the remnant merges with the ambient medium. 

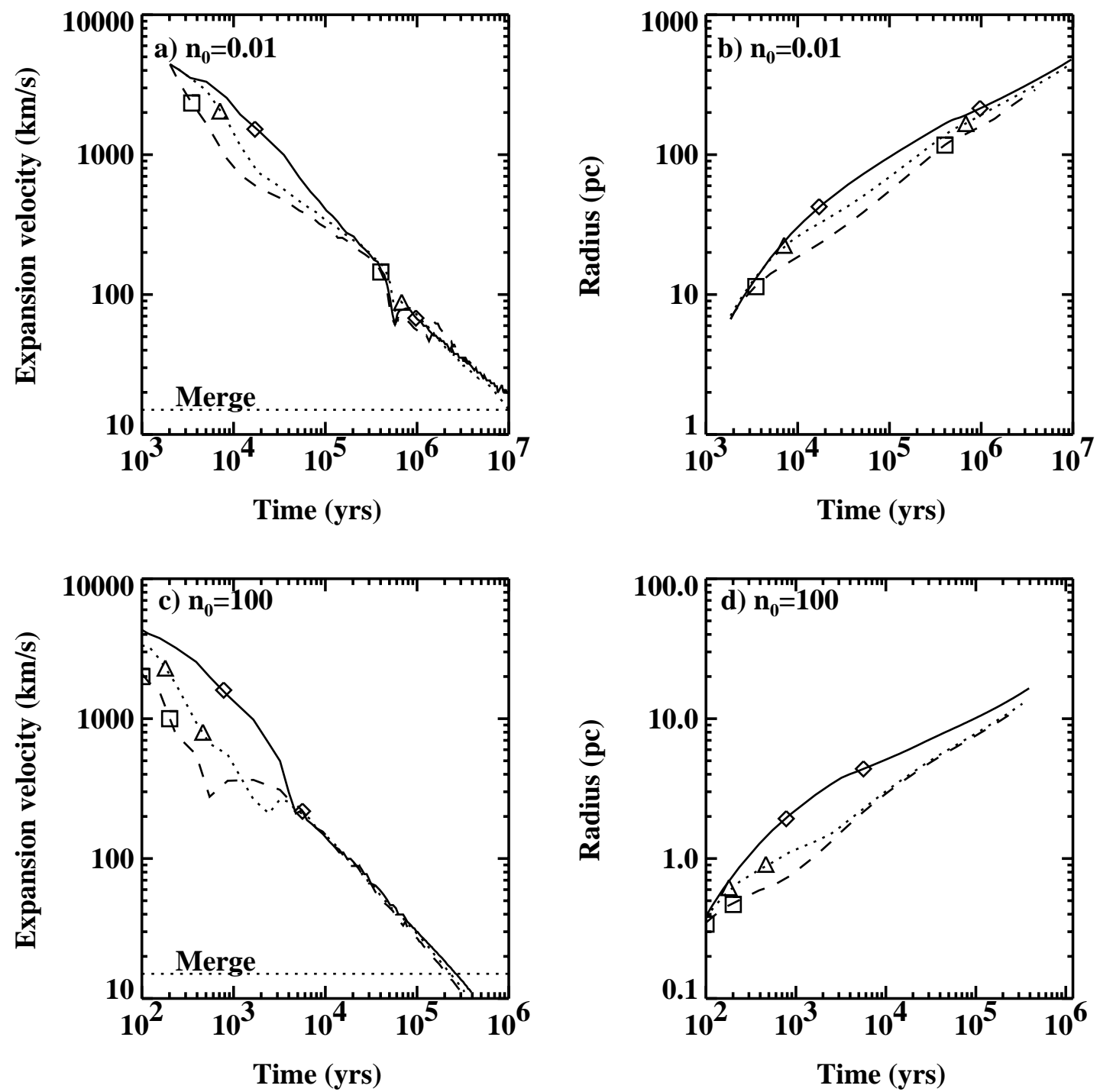

Fig. 1. Expansion velocity $\left(\mathrm{km} \mathrm{s}^{-1}\right)$ and radius $(\mathrm{pc})$ versus time (yrs) for remnants $\left(E=10^{51} \mathrm{erg}, M=10 M_{\odot}\right)$ expanding into an ambient density of $n_{0}=0.01 \mathrm{~cm}^{-3}$ and $n_{0}=100 \mathrm{~cm}^{-3}$. The temperature of the ambient medium is $10^{4} \mathrm{~K}$, and its sound speed is $15 \mathrm{~km} \mathrm{~s}^{-1}$. The solid line has $f^{\prime}=0$ (no mass loading), while the dotted and dashed lines have $f^{\prime}=3.16$ (substantial mass loading) and $f^{\prime}=31.6$ (high mass loading) respectively. The diamonds, triangles and squares indicate the end of the FE and QST stages for $f^{\prime}=0,3.16,31.6$ respectively.

This qualitative difference in the time-dependent behaviour of the mass loading between the conductively driven evaporation and ablation cases accounts for the differences in the evolutionary behaviour displayed in Fig. 1 of this paper and the evolution of the remnants discussed in Paper I. For the conductive case, the mass loading is extinguished at relatively early times and hence does not have much effect over the later stages of the remnant evolution. Once mass loading is "switched off" the ratio $M_{\text {load }} / M_{\text {swept }}$ depends solely on $M_{\text {swept }}$ and thus is not independent of $n_{0}$.

\subsection{Mass and energy fractions}

Figure 3 shows the time variation of the mass and energy fractions for $n_{0}=0.01 \mathrm{~cm}^{-3}$ and $f^{\prime}=0,3.16$ and 31.6. Although our initial conditions specify the remnant energy as being entirely kinetic at early times, a significant fraction is quickly thermalized.
Values of $f^{\prime}=3.16$ and 31.6 correspond to $f$ values of $10^{4}$ and $10^{5}$, respectively. These are much higher than the $f$ values discussed in Paper I. The reason for this choice is to approximately match the loaded to swept-up mass ratio in the two different mass loading scenarios at the onset of thin-shell formation, $t_{\text {sf }}$, i.e., $M_{\text {load }} / M_{\text {swept }} \approx 4$ in both cases. Since conductive evaporation is saturated only at early times in the remnant evolution (cf. Figs. 3c and 3e) and becomes negligible once average temperatures fall below $10^{6} \mathrm{~K}$, while hydrodynamic ablation becomes more important as the remnant's volume increases, obviously far higher values of $f$ are necessary in the conductive evaporation case to give $M_{\text {load }} / M_{\text {swept }} \approx 4$ at $t_{\text {sf }}$.

With $f^{\prime}=0$ (no mass loading) the thermal energy fraction peaks at approximately 0.72 i.e. the value for a Sedov-Taylor remnant expanding into a uniform medium (cf. Fig. 3b). Once the age of the remnant is comparable to $t_{\mathrm{sf}}$, the remnant begins to radiate its thermal energy, and the thermal energy fraction falls as the kinetic energy fraction rises. Mass injection speeds 

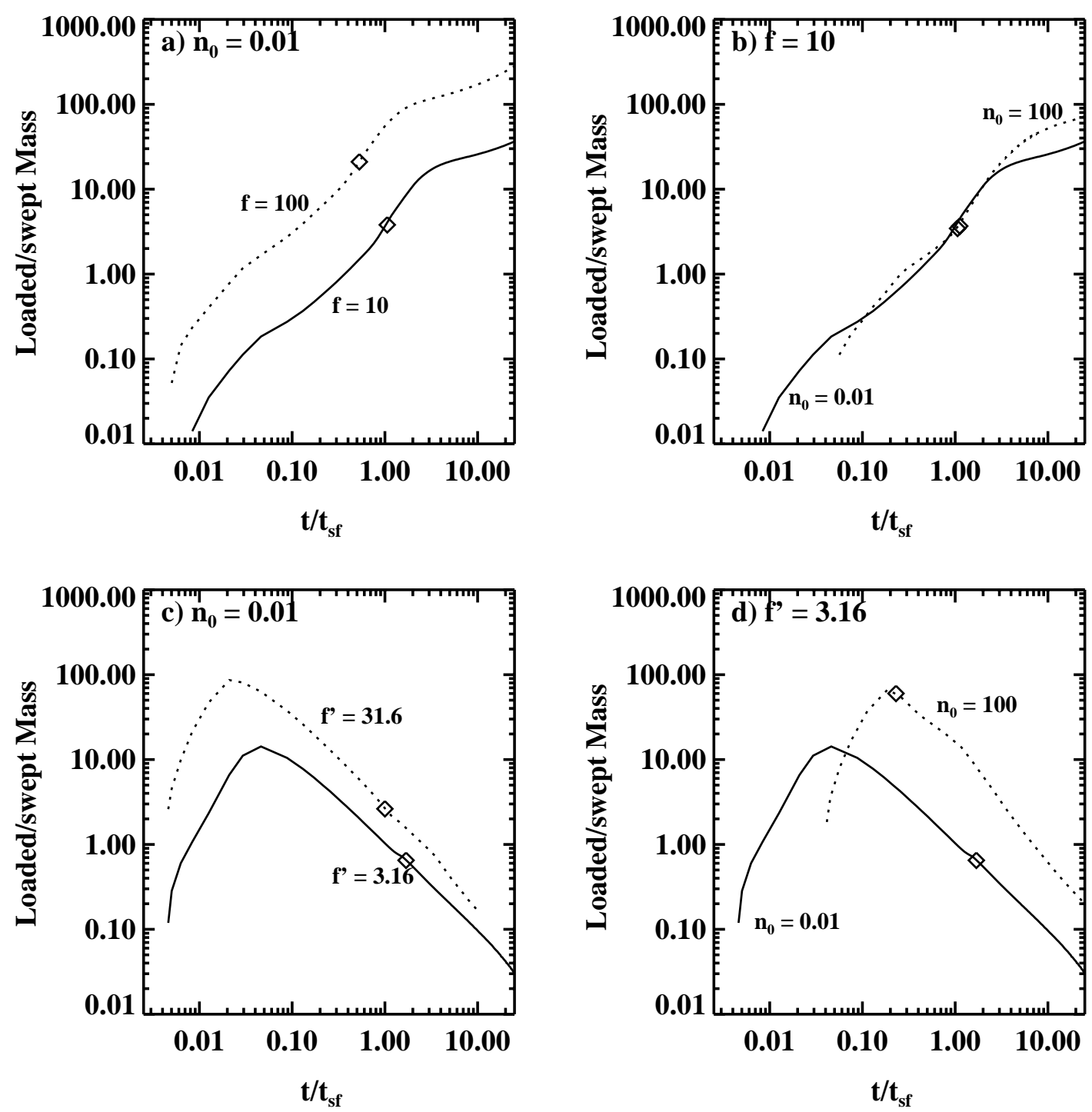

Fig. 2. The ratio of evaporated or ablated mass to swept-up mass as a function of time (normalized to the time of shell formation cf. Eq. (8)) for mass loading by hydrodynamic ablation (top panels) or by conductive evaporation (bottom panels). $t_{\mathrm{sf}}=4 \times 10^{5} \mathrm{yr}\left(n_{0}=0.01\right)$ and $2 \times 10^{3} \mathrm{yr}$ $\left(n_{0}=100\right)$. The diamonds mark the end of the QST stage, and almost overlap in b).

up these processes, and the thermal energy fraction first overshoots (see Sect. 3.1), then undershoots the Sedov-Taylor value (Figs. 3d, f). The decrease in the thermal energy fraction occurs not long after the end of the FE stage. This is long before radiative losses become significant, and the remnants are at this stage cooling by adiabatic expansion, such that thermal energy is converted into kinetic energy. This is despite the countering effect of mass loading, which at any given time tends to increase the thermal energy fraction relative to the kinetic energy fraction. However, we note that the thermal energy fraction begins to decline when the mass fraction of evaporated material starts to drop off and thus is simply a manifestation of the fact that mass loading is ceasing to be an important process in these remnants long before the onset of radiative cooling. In contrast, for the ablation cases discussed in Paper I, it was found that since mass loading becomes more important for the remnant as time goes on, the thermal energy fraction continues to increase right up until the end of the QST stage.
At very late times the energy in a SNR with mass loading due to conductive evaporation is predominantly kinetic, whereas in Paper I it was found that in the final stages of a SNR with mass loading due to ablation the continued mass loading ensures that thermal energy dominates.

\subsection{Evolution of physical quantities}

In Fig. 4 we show the density, pressure, temperature, and velocity distributions at specific times just before and after the predicted onset of thin-shell formation for models with $n_{0}=0.01$ and $f^{\prime}=0$ and $f^{\prime}=3.16$. The "structure" at small radii (where the ejected mass is) seen in the density and temperature plots is a consequence of the initial conditions and imposed spherical symmetry (which requires a reflection condition at $r=0$ ) but does not significantly affect global properties (see e.g. Cioffi et al. 1988). 

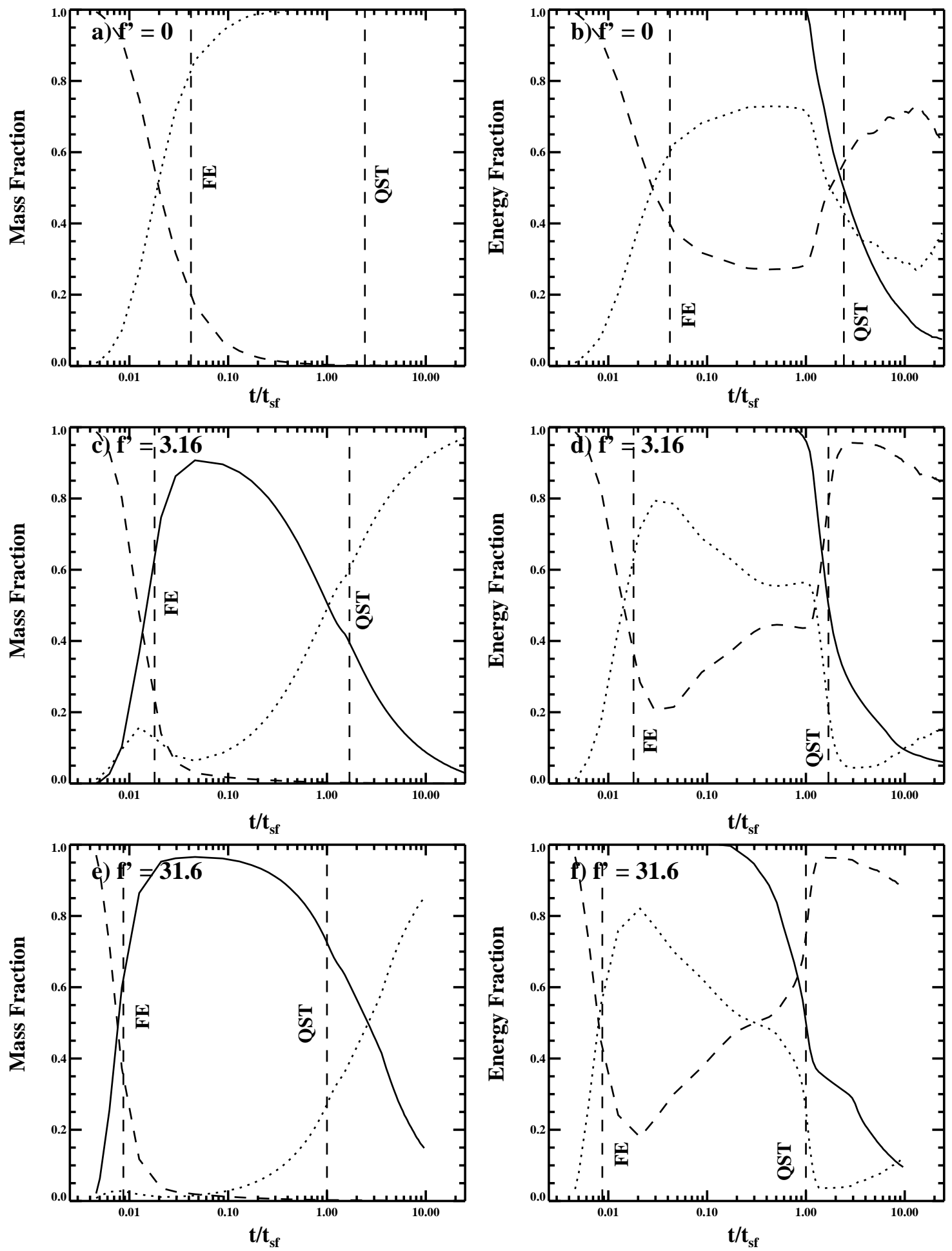

Fig. 3. Mass fractions (left panels) and energy fractions (right panels) as functions of the remnant age for $f^{\prime}=0,3.16,31.6\left(f=0,10^{4}, 10^{5}\right)$ and $n_{0}=0.01\left(t_{\mathrm{sf}}=4 \times 10^{5} \mathrm{yr}, E=10^{51} \mathrm{erg}, M=10 M_{\odot}\right)$. In each of the panels on the left the solid line shows the mass fraction of evaporated material, the dashed line shows the ejecta mass fraction, and the dotted line shows the swept-up mass fraction. In each of the panels on the right the dashed line shows the kinetic energy fraction, and the dotted line shows the thermal energy fraction, both in terms of the current remnant energy. The solid line shows the total energy as a fraction of the initial remnant energy. Note that we subtract the thermal energy swept-up by the remnant - if this is included both the total energy and the thermal energy fraction increase at late times. The vertical lines mark the ends of the free expansion (FE) and Quasi-Sedov-Taylor (QST) phases.

For $f^{\prime}=0$ the distributions of density etc. in the external part of the remnant (where the swept up mass is) correspond to the standard Sedov-Taylor solution (although, as in
Cioffi et al. 1988, at high ambient densities the remnant becomes radiative before fully entering this phase). The thin-shell formation time, $t_{\mathrm{sf}}$, for this ambient density is $4 \times 10^{5} \mathrm{yr}$ (from 

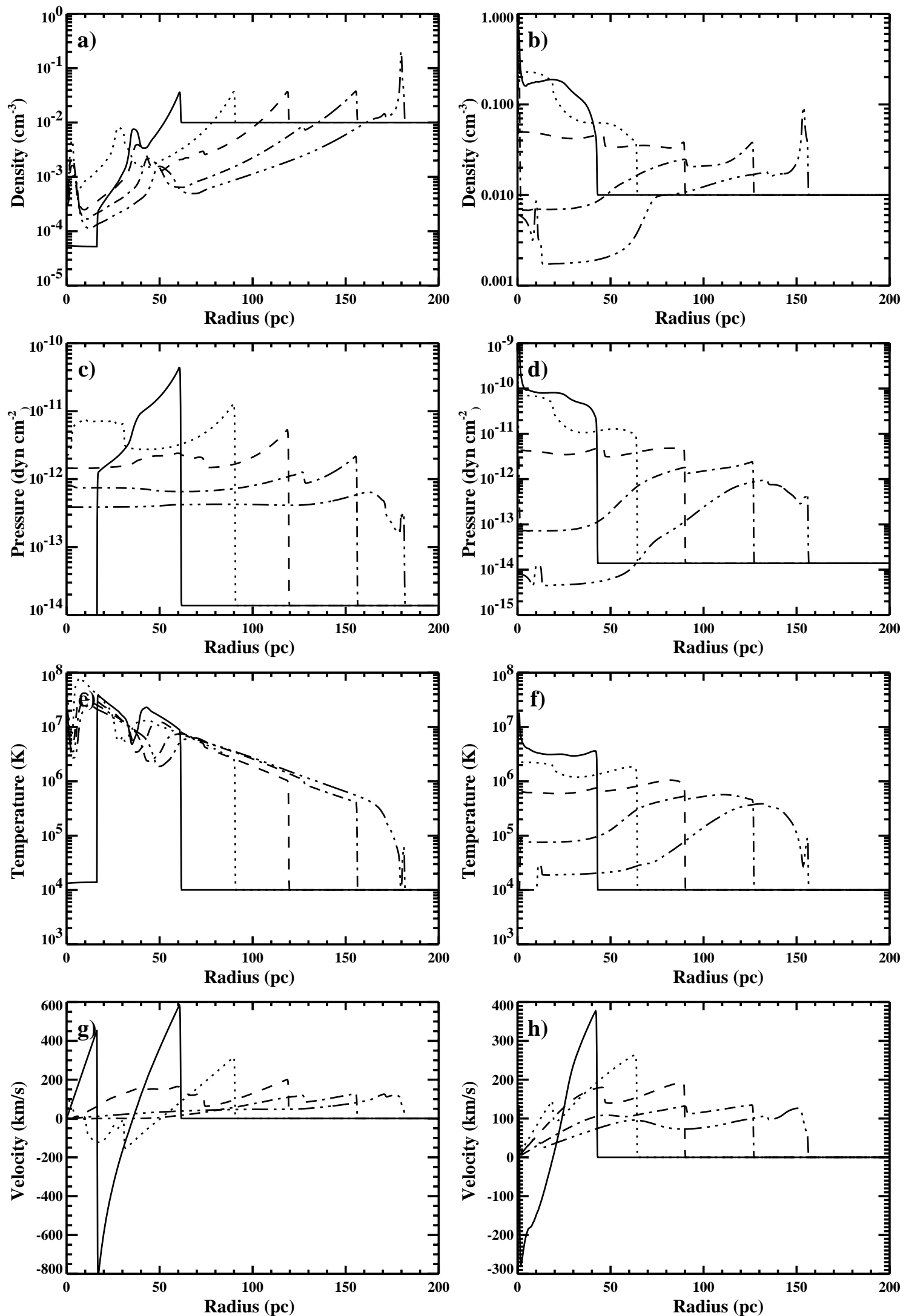

Fig. 4. Remnant evolution of density, pressure, temperature, and velocity as a function of age for $n_{0}=0.01$ and $f^{\prime}=0$ (left panels) or $f^{\prime}=3.16$ (right panels). The profiles are at time $t=3.54 \times 10^{4} \mathrm{yr}$ (solid); $t=8.59 \times 10^{4} \mathrm{yr}$ (dotted); $t=1.70 \times 10^{5} \mathrm{yr}\left(\right.$ dashed); $t=3.39 \times 10^{5} \mathrm{yr}$ (dot-dashed); $t=5.41 \times 10^{5} \mathrm{yr}$ (dot-dot-dot-dashed). $t_{\mathrm{sf}}=4 \times 10^{5} \mathrm{yr}$. 

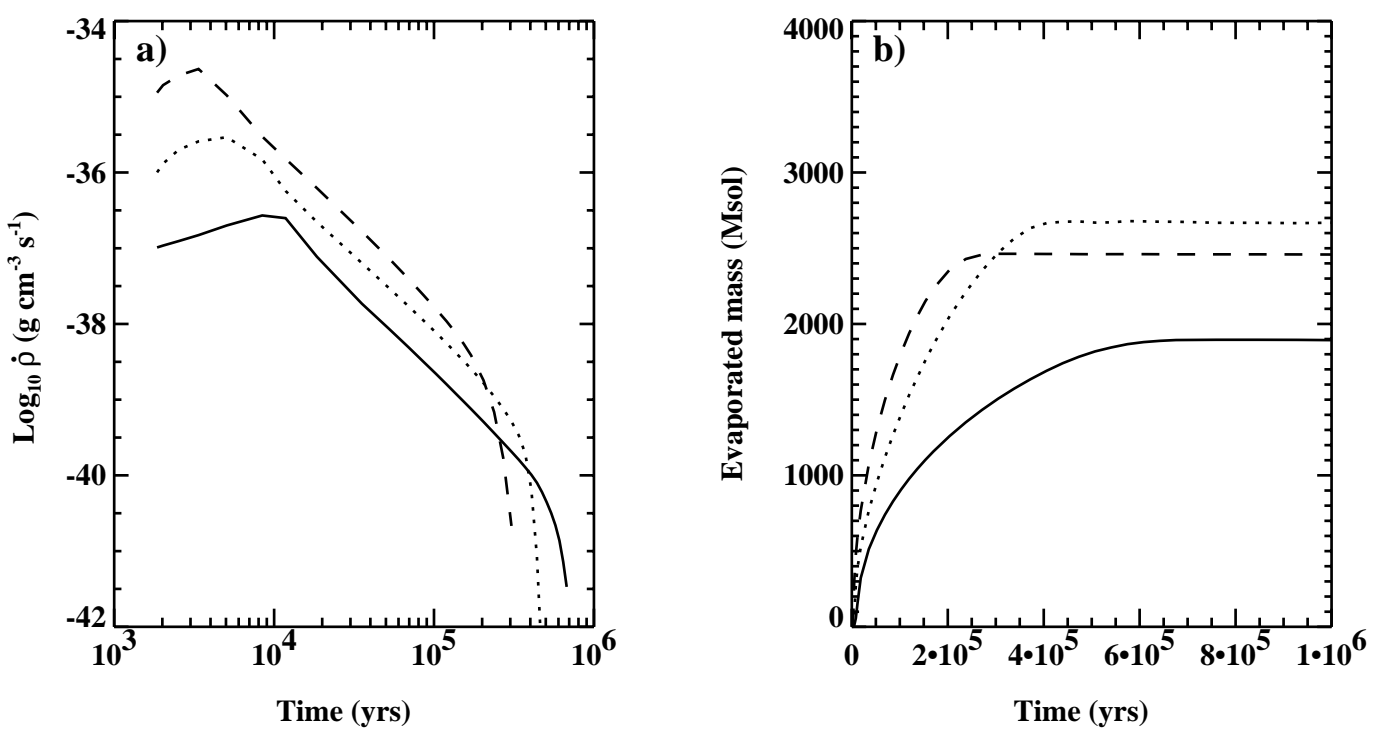

Fig. 5. Remnant averaged mass injection rate a), and integrated mass b), as a function of time for $n_{0}=0.01 \mathrm{~cm}^{-3}$ and $f^{\prime}=3.16$ (solid), $f^{\prime}=31.6$ (dotted), and $f^{\prime}=316$ (dashed). Here we define the remnant volume as a sphere with radius equal to that of the blast wave. The slight increase in $\dot{\rho}$ at early time results from the fact that the post-shock region where mass loading occurs is above the saturation temperature, while the volume filling factor simultaneously increases as the reverse shock propagates back to the centre of the remnant. Note also that the maximum amount of mass that can be evaporated when $n_{0}=0.01 \mathrm{~cm}^{-3}$ is $\lesssim 3000 M_{\odot}$.

Eq. (8)). Inspection of Fig. 4 (in particular, the pressure, which drops markedly in the region where strong radiative cooling starts) shows that our simulations are consistent with this value.

The addition of mass through the conductively driven evaporation of embedded clouds significantly alters the properties of the remnant, most obviously causing the interior density of the remnant to increase with respect to the no mass loading case. At early times, mass loading occurs mainly in the centre of the remnant (in the shocked ejecta region). Once temperatures here fall below $10^{6} \mathrm{~K}$ (due to adiabatic expansion), mass loading occurs mainly in the region of hot post-shock gas behind the blast wave, creating a "thick-shell" morphology (cf. Fig. 4b), resembling that of Cowie et al. (1981) and Dyson \& Hartquist (1987).

\subsection{Volume-averaged mass loading properties}

In Fig. 5a we show the remnant-averaged mass-injection rate (rate of mass evaporation divided by the volume of the remnant) as a function of time. At early times, the entire remnant is above $T=10^{7} \mathrm{~K}$, hence conductive evaporation is saturated and the remnant-averaged mass loading rate is approximately constant as the remnant expands (the small rise is caused by the increasing fractional volume of the hot shocked gas in the remnant). Once the average remnant temperature drops below $10^{7} \mathrm{~K}$ the mass evaporation rate is no longer saturated and $\dot{\rho}$, the rate of mass evaporation per unit volume, varies with time as $\dot{\rho} \propto t^{-2}$. This matches the behaviour found by Chièze $\&$ Lazareff (1981), who adopt $\dot{\rho} \propto T^{5 / 2}$, for heavily mass loaded remnants. This is a steeper dependence than the $\dot{\rho} \propto t^{-1}$ dependence found by White \& Long (1991), which is a consequence of their particular description for mass loading $\left(\dot{\rho} \propto T^{5 / 6}\right)$.

From Figs. 5b and 6 we see that the majority of the mass evaporation from the embedded clouds occurs after the average temperature of the remnant drops below $10^{7} \mathrm{~K}$. Once the average remnant temperature drops below $10^{5} \mathrm{~K}$ mass loading is effectively "switched off", the remnant averaged mass injection rate drops sharply, leaving the power-law dependence on time, and the quantity of evaporated mass remains constant in the remnant from this time onwards. Interestingly, this quantity does not depend linearly on the mass loading factor $f^{\prime}$ (Fig. 5b). Roughly half the evaporated mass is loaded after the average temperature has dropped below $10^{6} \mathrm{~K}$ for the model with $n_{0}=0.01 \mathrm{~cm}^{-3}$ and $f^{\prime}=3.16$. Hence our results should not be strongly affected by the precise value of the temperature $T_{\text {sat }}$ at which conductive evaporation becomes saturated, or by the initial conditions that we specify.

\subsection{End of the QST stage}

In Fig. 7, the ratio of the time at which the total energy of the remnant falls to one half of its initial energy, $t_{1 / 2}\left(f^{\prime}, n_{0}\right)$, to the shell formation timescale, $t_{\mathrm{sf}}\left(n_{0}\right)$, defined in Eq. (8), is shown as a function of the mass loading parameter $f^{\prime}$ for the five different values of the ambient density used. This figure shows far more variation with $n_{0}$ than the equivalent figure in Paper I, where the curve for the different values of the mass loading parameter lie on top of each other. In the conductively driven mass loading case the mass loading rate is sensitive to the thermal structure throughout the remnant, and radiative cooling, when it begins, occurs in a larger volume of gas (because of the "thick-shell" morphology). The radiative cooling rate thus depends on the density and temperature of the gas in this extended region, which have a non-simple relation to the mass loading factor $f^{\prime}$. In contrast, in the ablation cases discussed in Paper I, mass loading occurs in the narrow region just behind the blast wave, and so the amount of radiative cooling in this zone (which ultimately defines $t_{\mathrm{QST}}$ ) depends directly on the factor $f$. 


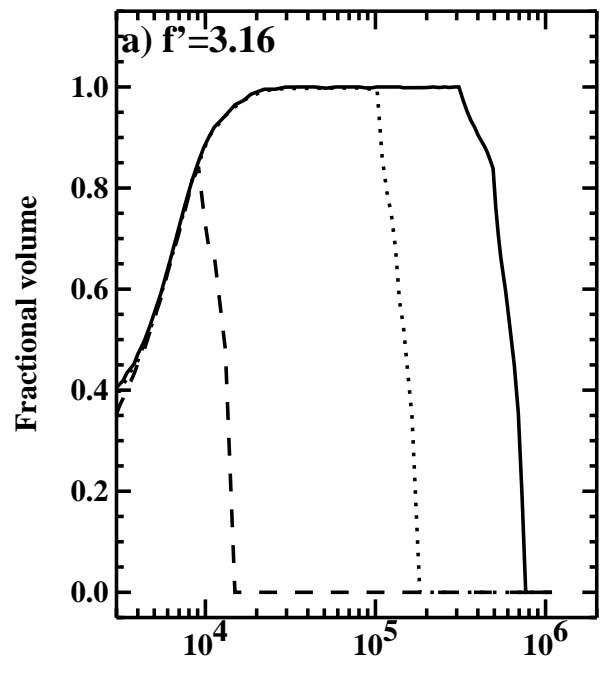

Time (yrs)

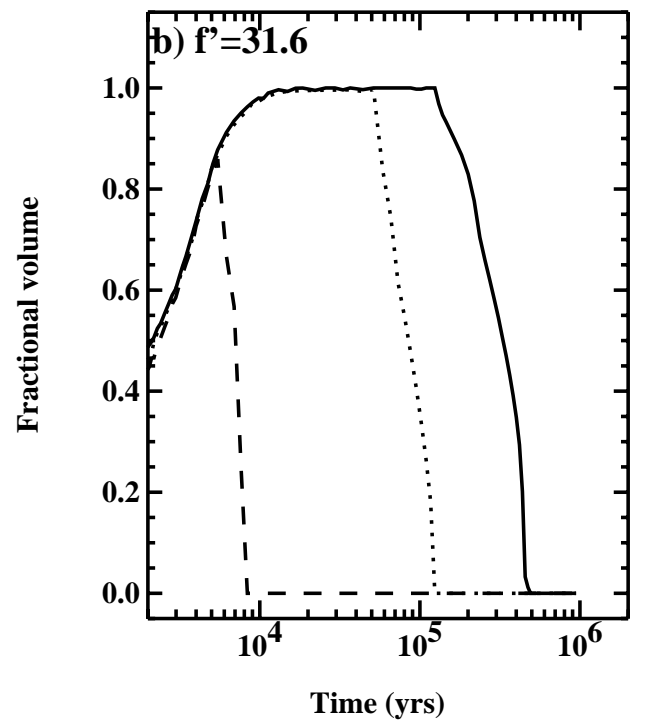

Fig. 6. The fractional volume of the remnant above a temperature of $10^{5} \mathrm{~K}$ (solid), $10^{6} \mathrm{~K}$ (dotted), and $10^{7} \mathrm{~K}$ (dashed) for $n_{0}=0.01 \mathrm{~cm}^{-3}$ and $f^{\prime}=3.16 \mathbf{a}$ ) or $f^{\prime}=31.6 \mathbf{b}$ ). At early times when the ejecta is being thermalized all of the shocked gas is hotter than $10^{7} \mathrm{~K}$ and the curves are coincident in each case, and rising towards a value of 1.0. When the mass loading is stronger the remnant cools quicker.

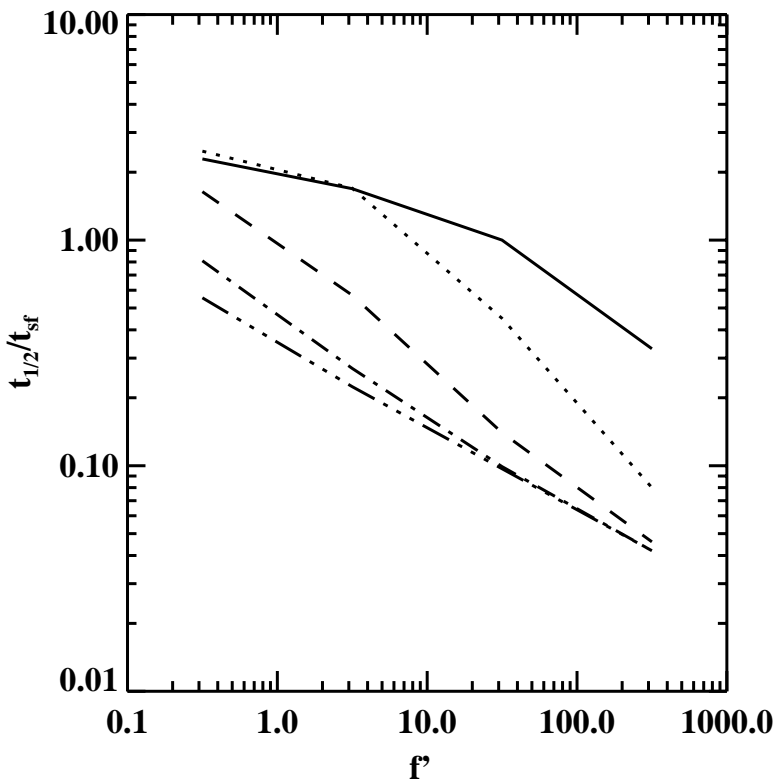

Fig. 7. Ratio of the half energy time to the thin shell formation time $\left(t_{1 / 2} / t_{\mathrm{sf}}\right)$ as a function of the mass loading parameter $f^{\prime}$ for the full range of intercloud ambient densities considered: $n_{0}=0.01$ (solid); $n_{0}=0.1$ (dotted); $n_{0}=1.0$ (dashed); $n_{0}=10.0$ (dot-dashed); $n_{0}=100.0$ (triple dot-dash).

\section{Discussion}

\subsection{The free expansion (FE) phase}

Table 1 gives the total mass in the remnant at the end of the FE phase, $M_{\mathrm{FE}}$, for remnants with an initial energy, $E=10^{51} \mathrm{erg}$, and ejecta mass, $M=10 M_{\odot}$. When there is no mass loading the total mass is independent of the ambient density (being approximately $56 M_{\odot}$, cf. Paper I), though with intermediate rates of mass loading (e.g. $\left.f^{\prime} \approx 0.316\right) M_{\mathrm{FE}}$ becomes dependent on $n_{0}$. For slightly higher mass loading rates $\left(f^{\prime} \geq 3.16\right)$
Table 1. The total mass, $M_{\mathrm{FE}}\left(M_{\odot}\right)$, in the remnant at the end of the free expansion stage for conductive mass loading. Apart from models with $f^{\prime}=0.316$, the mass is relatively invariant with $n_{0}$, being $\approx 56 M_{\odot}$ for $f^{\prime}=0$ and $\approx 37 M_{\odot}$ for $f^{\prime} \geq 3.16$. $E=10^{51} \mathrm{erg}, M=10 M_{\odot}$.

\begin{tabular}{rrrrrr}
\hline \hline & & & $f^{\prime}$ & & \\
$n_{0}$ & 0.0 & 0.316 & 3.16 & 31.6 & 316 \\
\hline 0.01 & 56.4 & 45.6 & 38.1 & 36.2 & 36.4 \\
0.10 & 56.4 & 43.3 & 37.4 & 36.9 & 37.6 \\
1.00 & 56.0 & 42.4 & 37.6 & 36.2 & 36.2 \\
10.0 & 55.6 & 41.6 & 36.9 & 37.6 & 37.6 \\
100.00 & 56.0 & 39.3 & 37.6 & 37.0 & - \\
\hline
\end{tabular}

$M_{\mathrm{FE}} \approx 37 M_{\odot}$, and is insensitive to both $f^{\prime}$ and $n_{0}$. The fact that $M_{\mathrm{FE}}$ is dependent on $f^{\prime}$ for a given $n_{0}$ is consistent with Figs. 3b, d, f, where it can be seen that the remnant energy thermalizes more rapidly with increasing $f^{\prime}$. This behaviour arises from the fact that mass loading reduces the rate at which the remnant expands at early times, as it causes more braking in the ejecta and sends the reverse shock back through it more quickly. In contrast, we note that $M_{\mathrm{FE}}$ is essentially constant when mass loading occurs by hydrodynamic ablation (Paper I). Since the mass loading is essentially saturated in both formulations at this stage, these differences arise from the fact that in this paper we also mass load in the shocked ejecta (unlike in Paper I), which has the effect of increasing its pressure.

In Fig. 8 we show the radius at the end of the free-expansion stage, $R_{\mathrm{FE}}$, as a function of $n_{0}$ and $f^{\prime}$. We derive an appropriate analytical approximation for the dependence of $R_{\mathrm{FE}}$ on $n_{0}$ and $f^{\prime}$ by following the same procedure as in Sect. 4.1 of Paper I. The agreement between the simulations and the analytical approximation is generally good, although the latter systematically underestimates $R_{\mathrm{FE}}$ at high values of $f^{\prime}$ (as was also found in Paper I, where an explanation for this is given). For future purposes, however, we are more concerned with accurate 


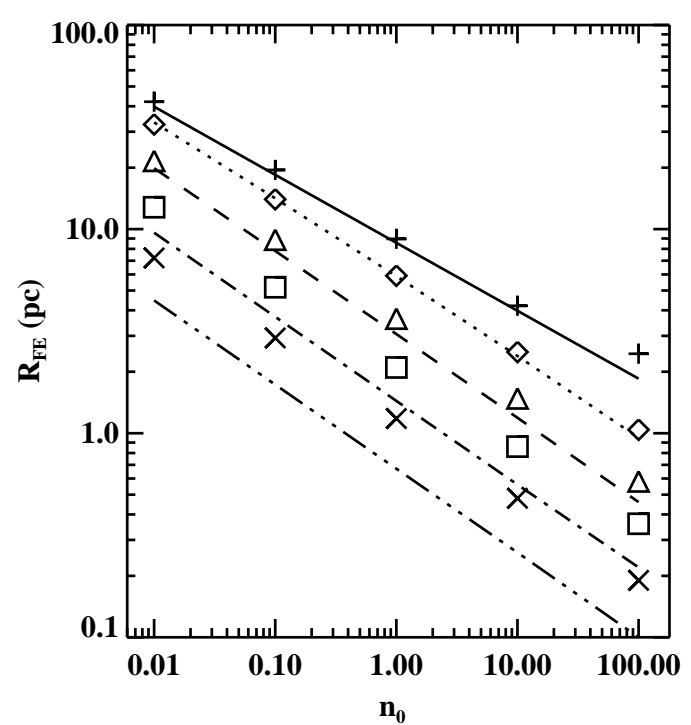

Fig. 8. Radius at the end of the free expansion stage as a function of ambient density. The symbols represent the results of the numerical simulations and the lines are from Eqs. (8) and (9) in Paper I, appropriately modified for $M_{\mathrm{FE} \text {,avg }} \approx 47 M_{\odot}$, and using the relation $f^{\prime}=316 f$. The symbols and lines are: $f^{\prime}=0.0$ (plus, solid); $f^{\prime}=0.316$ (diamond, dotted); $f^{\prime}=3.16$ (triangle, dashed); $f^{\prime}=31.6$ (square, dot-dashed); $f^{\prime}=316$ (cross, triple dot-dashed).

estimates for $R_{\mathrm{QST}}$, the radius at the end of the Quasi-SedovTaylor phase. We note that the much higher values of $f$ which we consider in this paper lead to a greater range in $R_{\mathrm{FE}}$ at a given $n_{0}$.

\subsection{The Quasi-Sedov-Taylor (QST) phase}

In Fig. 9 and Table 2 we show the variation of $R_{\mathrm{QST}}$ with $n_{0}$ and $f^{\prime}$. We find that the expression

$R_{\mathrm{QST}}\left(f^{\prime}, n_{0}\right) \approx 0.75 R_{\mathrm{QST}}\left(f^{\prime}=0\right) f^{\prime-0.05} 10^{-b \tanh \left(n_{0} / a\right)}$,

where $a=6 \times 10^{-0.9\left(1-\mathrm{e}^{-x}\right) x}, b=0.17\left(\log _{10} f^{\prime}+2.5\right)^{1.1}$, and $x=$ $\log _{10} f^{\prime}+0.5$ achieves a good fit to the numerical results. We plot values of $R_{\mathrm{QST}}$ using Eq. (12) in Fig. 9.

The ratio of the radius at the end of the Quasi-Sedov-Taylor phase to the radius at the same stage for the case where $f^{\prime}=0$ $\left(R_{\mathrm{QST}} / R_{\mathrm{QST}}\left(f^{\prime}=0\right)\right)$ is shown in Fig. 10 for all $f^{\prime}$ and $n_{0}$. Although there is variation with $n_{0}$ for $n_{0} \leq 10$, this ratio appears to become fairly insensitive to $n_{0}$ for larger values of $f^{\prime}$. We further note that the behaviour seen in Figs. 7 and 10 is qualitatively similar (which is also the case for ablative mass loading cf. Paper I).

For an ambient density, $n_{0}=0.01$, the approximation noted in Eq. (11) of Paper I, with substitution of $f$ with $f^{\prime}$, i.e.

$R_{\mathrm{QST}} \approx 0.9 R_{\mathrm{QST}}\left(f^{\prime}=0\right)\left(10 \sqrt{f^{\prime}}\right)^{-0.09 \log _{10} f^{\prime}}$,

is a good fit, while for high ambient densities $\left(n_{0} \sim 100.0\right)$ an excellent approximation is

$\log _{10}\left[R_{\mathrm{QST}} / R_{\mathrm{QST}}\left(f^{\prime}=0\right)\right] \approx-0.3 \log _{10} f^{\prime}-0.5$.

In the equations in this section it is again assumed that the remnants have an initial energy, $E=10^{51} \mathrm{erg}$, and ejecta mass, $M=10 M_{\odot}$.

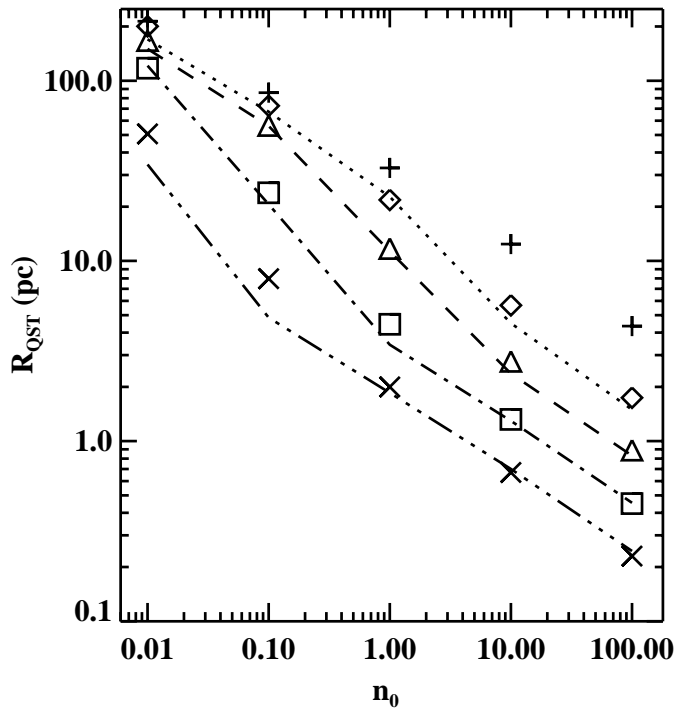

Fig. 9. Radius at the end of the Quasi-Sedov-Taylor stage as a function of $n_{0}$ and $f^{\prime}$. The symbols represent the results of the numerical simulations and the lines are the analytic approximation of Eq. (12), and denote $f^{\prime}=0.0$ (plus); $f^{\prime}=0.316$ (diamond, dotted); $f^{\prime}=3.16$ (triangle, dashed); $f^{\prime}=31.6$ (square, dot-dashed); $f^{\prime}=316$ (cross, triple dot-dashed).

Table 2. The radius (pc) of a conductively mass loaded remnant at the end of the Quasi-Sedov-Taylor stage. $E=10^{51} \mathrm{erg}, M=10 M_{\odot}$.

\begin{tabular}{rrrrrr}
\hline \hline & & \multicolumn{1}{c}{$f^{\prime}$} \\
$n_{0}$ & 0.0 & 0.316 & 3.16 & 31.6 & 316 \\
\hline 0.01 & 214 & 201 & 167 & 117 & 50.7 \\
0.10 & 85.8 & 72.6 & 56.4 & 23.9 & 7.97 \\
1.00 & 32.8 & 21.8 & 11.7 & 4.45 & 2.00 \\
10.0 & 12.4 & 5.67 & 2.77 & 1.32 & 0.67 \\
100.00 & 4.35 & 1.74 & 0.89 & 0.45 & 0.23 \\
\hline
\end{tabular}

\section{Conclusions}

We have extended work presented in Paper I on the evolution of mass loaded supernova remnants by considering mass loading by conductively driven evaporation of embedded clouds. Paper I confirmed that mass injection can strongly influence remnant evolution, and we also establish here that the nature of the mass injection process is also important in this regard. This is due to the fact that mass loading through conductive evaporation is extinguished at relatively early times. Hence, conductively driven mass loading does not appreciably alter the later stages of remnant evolution, when the remnant is dominated by swept up ambient gas. Therefore remnants that ablatively mass load are dominated by loaded mass and thermal energy at late times (Paper I), while those that conductively mass load are dominated by swept-up mass and kinetic energy. The greater dominance of loaded mass in the ablative case means that such remnants evolve more quickly, and reach all dynamical stages earlier. At a given age they tend to be both more massive and smaller than equivalent remnants which are conductively mass loaded. 


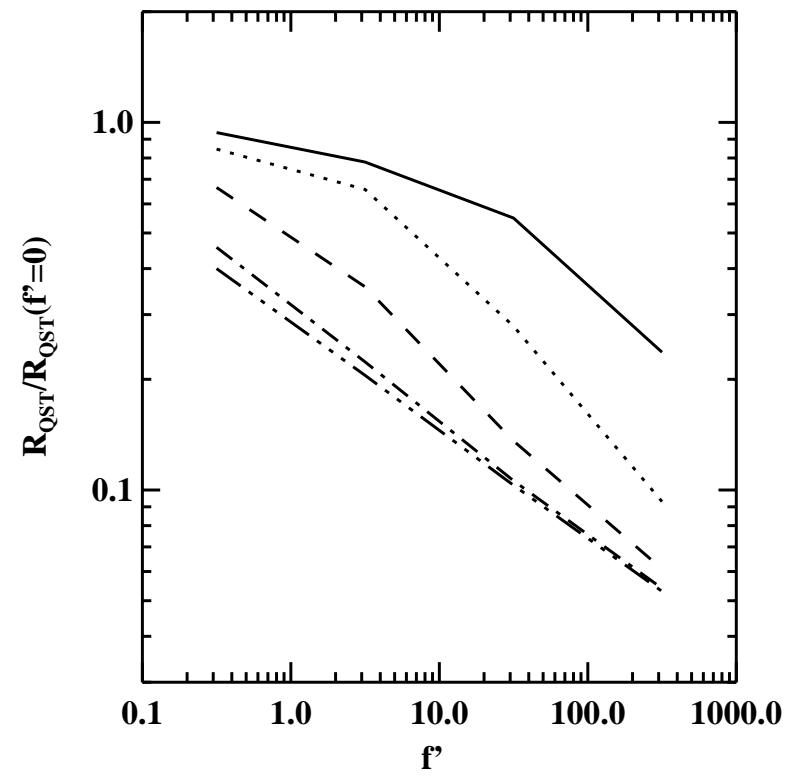

Fig. 10. Ratio of the radius at the end of the Quasi-Sedov-Taylor phase to the radius at the same stage for the case where $f^{\prime}=0$ $\left(R_{\mathrm{QST}} / R_{\mathrm{QST}}\left(f^{\prime}=0\right)\right)$ against $f^{\prime}$ for all $n_{0}: n_{0}=0.01$ (solid); $n_{0}=0.1$ (dotted); $n_{0}=1.0$ (dashed); $n_{0}=10.0$ (dot-dashed); $n_{0}=100.0$ (triple dot-dashed).

We are able to confirm some of the properties of conductively mass loaded remnants predicted from self-similar solutions, and in particular find that such remnants may display a thick-shell morphology (cf. the hydrodynamic results presented in Cowie et al. 1981 and the similarity solutions presented in Dyson \& Hartquist 1987).

In this work we have been particularly interested in the range of conductively mass loaded supernova remnants at the time at which they have radiated away half of their initial energy (see Table 2). It was noted in Sect. 2 that $f^{\prime}$ may be dependent on $n_{0}$. This behaviour would pick-out a roughly diagonal line in Table 2, and implies that the radius of remnants at the end of the quasi-Sedov-Taylor stage has less variance with $n_{0}$ than would otherwise be the case. However, since $f^{\prime}$ is also dependent on the number density of cold clouds we do not expect a particularly tight relationship.

Simple approximations that fit the evolution of the range of supernova remnants which conductively mass load, and which are complementary to similar approximations in Paper I, have also been found. In both works it is assumed that the remnant has an initial energy, $E=10^{51} \mathrm{erg}$, and an ejecta mass, $M=10 M_{\odot}$. We expect that the evolution of remnants which ablatively mass load will be fairly insensitive to the progenitor mass, because the majority of the mass loading occurs after the FE stage ends, at which point the swept up mass is about 6 times greater than the progenitor mass. In the conduction case it is not so clear what will happen, because most of the mass loading occurs in the early stages when the remnant is becoming thermalized. Furthermore, the mass loading may well be more dependent on our model assumptions (such as the saturation temperature, $T_{\mathrm{sat}}$ ), than on the progenitor mass, at least in some regions of parameter space. With regards to the possibility of different explosion energies, we note that our solutions should scale in a similar way to the time of thin shell formation, $t_{\text {sf }} \propto E^{3 / 14}$ (cf. Eq. (8)).

Our range approximations will form the basis of future work to investigate galactic superwinds formed by the combination of many overlapping supernovae.

Acknowledgements. We would like to thank Dr. R. Bandiera for constructive comments which led to clarification of our assumptions and generally improved the paper. JMP would also like to thank PPARC for the funding of a PDRA position. This work has made use of NASA's Astrophysics Data System Abstract Service.

\section{References}

Arthur, S. J., \& Falle, S. A. E. G. 1991, MNRAS, 251, 93

Arthur, S. J., \& Falle, S. A. E. G. 1993, MNRAS, 261, 681

Arthur, S. J., \& Henney, W. J. 1996, ApJ, 457, 752

Chevalier, R. A., \& Blondin, J. M. 1995, ApJ, 444, 312

Chièze, J. P., \& Lazareff, B. 1981, A\&A, 95, 194

Chu, Y.-H., et al.1999, New Views of the Magellanic Clouds, ed. Y.-H. Chu, N. Suntzeff, J. Hesser, \& D. Bohlender, IAU Symp., 190, 143

Cioffi, D. F., McKee, C. F., \& Bertschinger, E. 1988, ApJ, 334, 252

Cowie, L. L., \& McKee, C. F., ApJ, 211, 135

Cowie, L. L., McKee, C. F., \& Ostriker, J. P. 1981, ApJ, 247, 908

Cowie, L. L., \& Songalia, A. 1977, Nature, 266, 501

Dyson, J. E., Arthur, S. J., \& Hartquist, T. W. 2002, A\&A, 390, 1063 (Paper I)

Dyson, J. E., \& Hartquist, T. W. 1987, MNRAS, 228, 453

Falle, S. A. E. G., \& Garlick, A. R. 1982, MNRAS, 201, 635

Falle, S. A. E. G., \& Komissarov, S. S. 1996, MNRAS, 278, 586

Falle, S. A. E. G., \& Komissarov, S. S. 1998, MNRAS, 297, 265

Franco, J., Miller, W. W., Arthur, S. J., Tenorio-Tagle, G., \& Terlevich, R. 1994, ApJ, 435, 805

Franco, J., Tenorio-Tagle, G., Bodenheimer, P., \& Rozyczka, M. 1991, PASP, 103, 803

Gull, S. F. 1973, MNRAS, 161, 47

Hartquist, T. W., Dyson, J. E., Pettini, M., \& Smith, L. J. 1986, MNRAS, 221, 715

McKee, C. F., \& Cowie, L. L. 1977, ApJ, 215, 213

McKee, C. F., \& Ostriker, J. P. 1977, ApJ, 218, 148

Pittard, J. M., Dyson, J. E., Falle, S. A. E. G., \& Hartquist, T. W. 2001, A\&A, 375, 827

Suchkov, A. A., Berman, V. G., Heckman, T. M., \& Balsara, D. S. 1996, ApJ, 463, 528

Terlevich, R., Tenorio-Tagle, G., Franco, J., \& Melnick, J. 1992, MNRAS, 255, 713

Truelove, J. K., \& McKee, C. F. 1999, ApJS, 120, 299

Warren, J. S., Hughes, J. P., \& Slane, P. O. 2003, ApJ, 583, 260

White, R. L., \& Long, K. S. 1991, ApJ, 373, 543 